\title{
Manifestation of deformation effect in band spectra in crystals with inhomogeneous bonding
}

\author{
D.M.Bercha ${ }^{1,2}$, K.Z.Rushchanskii ${ }^{1}$, I.V.Slipukhina ${ }^{1}$, \\ I.V.Bercha ${ }^{1}$ \\ 1 Uzhgorod National University, \\ 54 Voloshyn Str., 88000 Uzhgorod, Ukraine, \\ 2 Institute of Physics, Pedagogical University, \\ 16a Rejtana Str., 35-310 Rzeszów, Poland
}

Received October 25, 2002, in final form April 3, 2003

\begin{abstract}
The effect of the shear strains on the energy spectrum of the strongly anisotropic SbSI crystal has been investigated by group-theoretical method in combination with the Pikus method of invariants. The first-principles local density approximation has been implemented to determine the band structure of the crystal. Ab initio calculations of the band structure have shown an exact localization of twofold degenerate maximum of the valence band in the T point. It turned out that the shear strains result in the band topology changes in the vicinity of the $\mathrm{T}$ point and the $\mathrm{k}$-linear term appears in the corresponding dispersion law.
\end{abstract}

Key words: ab initio, deformations, SbSI

PACS: 71.15.Ap, 71.15.Hx, 71.15.Mb, 61.50.Ah

The inhomogeneous bonding in the strongly anisotropic crystals with a complicated unit cell results in some peculiarities of their energy band structure caused by external effect. Such crystals are the crystals with distinguished structural units in the form of layers or chains. Some of them belong to the group of ferroelectricssemiconductors. The SbSI crystal is one of the representatives of the mentioned group.

As it is well known, the main characteristic of semiconductors is their energy spectrum. The changes in the energy spectrum under external effects are crucial in the manifestation of such effects on the electronic physical properties. One of the above mentioned factors is the effect of external strains.

The kp-theory and its modern modifications make it possible to investigate dispersion laws of electrons and holes for strained and unstrained crystals. Nevertheless,

*E-mail: iv@iss.univ.uzhgorod.ua 
taking into account the symmetry only, we cannot define the points of absolute band extrema. For the clarification of the situation it is necessary to carry out the band structure calculations focusing a special attention on the vicinity of the points, in which the group-theoretical analysis permits the existence of the extrema in $E(\mathbf{k})$ dependence.

There exist several numerical calculations of the energy band structure of the SbSI crystal with the symmetry of $D_{2 h}^{16}$ (Pnam) space group [1-3]. Because of the ambiguity of these results we carried out our own calculations of the band structure of the SbSI crystal in the paraelectric phase. It should be noted that a more convenient coordinate system to describe the SbSI crystal is that of the Pnam group, since this crystal exhibits a phase transition to the ferroelectric phase describable in terms of the Pna2 $\left(C_{2 v}^{9}\right)$ space group [4], which is a subgroup of the Pnam group. There are twelve atoms in the unit cell of the SbSI crystal which create two translationally nonequivalent chains. Lattice constants of this crystal in the coordinate system of the Pnam group are as follows: $a_{1}=8.52 \AA, a_{2}=10.13 \AA, a_{3}=4.10 \AA$. The atomic coordinates are taken from [5].

The band structure of the SbSI crystal was obtained by ab initio calculations. The non-local norm-conserving pseudopotentials [6] were used, calculated on the basis of the Hammann scheme [7] and implemented in the package program fhig8PP [8]. In order to take into account the exchange-correlation interaction, the results obtained by Caperley and Alder in the local approximation [9] were implemented in the project. Perdew and Zunger's [10] analytic fit to these results was used in our calculations. Scalar relativistic effects were included in the pseudopotentials. The selected parameters ensure the generation of the "ghost-free" pseudopotentials [11]. The band structure of the SbSI crystal was calculated by means of the fhi98md program [12]. The wave functions were expanded in plane waves with kinetic energy up to $20 \mathrm{Ry}$, which corresponds to approximately 3700 plane waves. To obtain the ground state of the electron system, the Joannopoulos algorithm [12] was used in the calculations. The special k-points method [13] was implemented to carry out the integration in the $\mathbf{k}$ space over the Brillouin zone (BZ). The 105 special $\mathbf{k}$ points were selected in the irreducible part of the BZ to calculate the ground state of the SbSI crystal. The chosen parameters enabled us to obtain a good convergence in calculations. We did not take into account the spin-orbit interaction.

The band structure of the SbSI crystal is shown in figure 1. Since the SbSI crystal is strongly anisotropic, in the $\mathrm{Z}-\mathrm{T}$ and $\mathrm{R}-\mathrm{U}$ directions a small dispersion of the branches is noticeable, especially for the deep branches of the valence band. The minimum of the conduction band is located in the $\mathrm{S}$ point. This result coincides with the data noted in $[3,14]$ when the change in the coordinate system is introduced. It should be noted that the aforementioned band structure of the SbSI crystal was determined by the semi-empirical pseudopotential method. In the spectrum presented in figure 1 one can see a small splitting of branches in the $T$ point caused by a weak interaction between the chains of the crystal. This behavior is predictable [15]. The width of the obtained indirect band gap is equal to $1.5 \mathrm{eV}$, while the experimental estimation of this value yields $E_{\text {gmin }} \sim 1.8 \mathrm{eV}$ [4]. The most significant discrepancy 

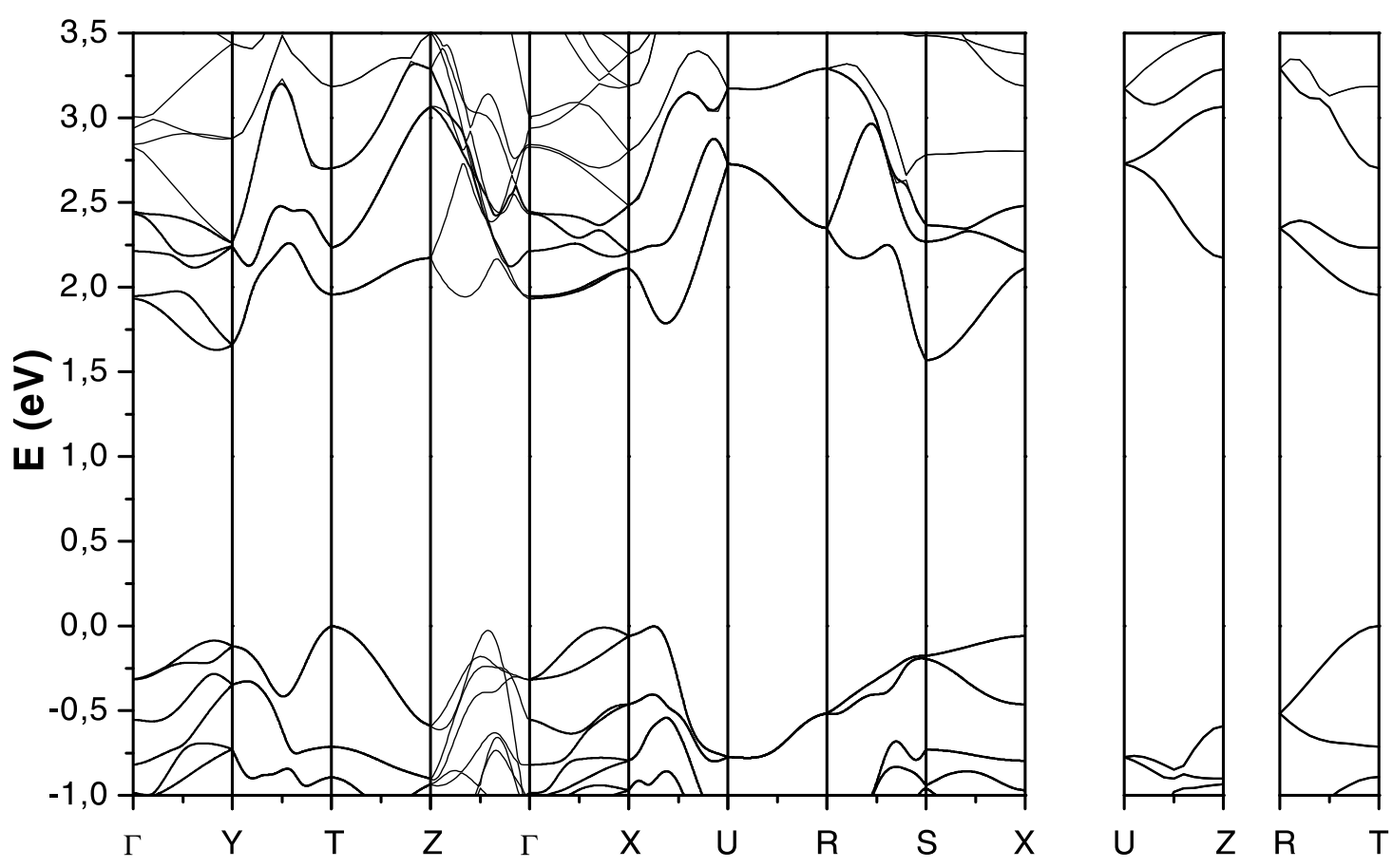

Figure 1. The energy band structure of the SbSI crystal.

between the band structure of the SbSI crystal obtained in the semi-empirical pseudopotential calculations and that obtained by ab initio calculations can be specified as follows. The maximum of the valence band is not located in the $\Gamma$ point, as it is obtained in the calculations done by the semi-empirical pseudopotential method. The distinct maxima of the valence band are observable along the $\Gamma-\mathrm{Z}, \Gamma-\mathrm{Y}, \Gamma-\mathrm{X}$ directions as well as in the $\mathrm{T}$ point. It is evident that for low-energy values in the valence band there exist nine separate complexes consisting of 4 branches. Moreover, their symmetry in the $\Gamma$ point is the same as the one calculated on the basis of the empty-lattice approximation. In the $\Gamma-\mathrm{Z}$ direction an interaction between the branches can be found.

There are two translationally nonequivalent chains in the unit cell of the SbSI crystal and this causes Davidov's splitting over the Brillouin zone between some energy bands. This splitting was simulated and investigated in [15]. As one can see from the results obtained in this paper, the splitting should be relatively smaller in the $\mathrm{T}$ point than the one in other directions of the Brillouin zone. Our numerical calculations showed that in the minimal band complex, consisting of four subbands, located in the depth of the valence band, such a splitting really exists in the $\mathrm{T}$ point and it is a minimal one. The $\mathrm{T}$ point is one of the extrema of the valence band and it also presents interest because, as it will be shown a little later in this paper, the twofold degenerate dispersion dependence in $\Gamma-Y$ and $\Gamma-Z$ directions does not 
split under the shear strains in the $\mathrm{T}$ point itself and turns into the dependence containing a k-linear term in the vicinity of the $\mathrm{T}$ point. To show this we shall here use the Pikus method of invariants [16] to obtain the dispersion law $E(\mathbf{k})$ in the vicinity of the $\mathrm{T}$ point for the strained and unstrained SbSI crystal.

It should be noted that, as it was mentioned above at the very beginning of this paper, there is an inhomogeneous bonding in the SbSI crystal (weak between chains and strong between atoms of chains). It results in the symmetry lowering of the simulated crystal comparatively with the symmetry of the unstrained crystal because of the mathematical deformation which is necessary for the reconstruction of the boundary conditions broken by applying the external strains [17]. The easiest way to get sure that the $\mathbf{k}$-linear terms appear under the shear strains is to compare the extremeness of the corresponding dispersion curves along the main directions of the Brillouin zone of the unstrained and simulated crystals (table 1). The latter are described by different space groups. It turned out [17], that double degeneracy does not disappear in the $\mathrm{T}$ point under the shear strain $\varepsilon_{x y}\left(\varepsilon_{x z}\right)$ and in the corresponding dispersion dependence a $\mathbf{k}$-linear term appears in $k_{z}$ direction under the $\varepsilon_{x y}$ strain and in $k_{y}$ direction under the $\varepsilon_{x z}$ strain.

Now let us show that the same result can be obtained by the Pikus method of invariants modified by accounting for the terms of higher than $\mathbf{k}^{2}$ order of magnitude when we neglect the layered character of the crystalline structure. So, we shall consider the simulated crystal after the mathematical deformation which is described by the same space group as the unstrained one.

As it is well known, a dispersion law $E(\mathbf{k})$ in the Pikus method can be obtained by solving a secular equation $\left|D_{i j}(\mathbf{k}, \tilde{\varepsilon})-E(\mathbf{k}) \delta_{i j}\right|=0$, where

$$
D(\mathbf{k}, \tilde{\varepsilon})=\sum_{r, s} C_{s}^{r} \sum_{i} A_{i s} f_{i s}^{r}(\mathbf{k}, \tilde{\varepsilon}) \text {, }
$$

and $A_{i s}$ are linearly independent matrices transforming according to $\tau^{s}(g)$ representation; $f_{i s}^{r}(\mathbf{k}, \tilde{\varepsilon})$ are polynomials of the components of $\mathbf{k}$ and tensor of deformation $\tilde{\varepsilon}$. These polynomials are transformed according to $\tau^{s *}(g)$ representation (star means a complex conjugation). The $\tau^{s}(g)$ is the representation of the group of the wave vector $\mathbf{k}=0$ [16] and is defined in different ways depending on the cases a), b), or c) according to the Herring classification for the representations of the wave vector group in a chosen special point in the Brillouin zone. The matrices $A_{i s}$ and the basis functions $f_{i s}^{r}(\mathbf{k}, \tilde{\varepsilon})$ for the $\mathrm{T}$ point in the presence of the shear strains $\varepsilon_{x y}\left(\varepsilon_{x z}\right)$ are presented in table 2 .

In table $2, f= \pm 1$ defines the parity of the $f_{i s}^{r}(\mathbf{k}, \tilde{\varepsilon})$ functions. The $D(\mathbf{k})$ matrix for the unstrained crystal is defined as follows:

$$
D(\mathbf{k})=\left(\begin{array}{ll}
a k_{x}^{2}+b k_{y}^{2}+c k_{z}^{2} & d k_{y} k_{z} \\
d k_{y} k_{z} & a k_{x}^{2}+b k_{y}^{2}+c k_{z}^{2}
\end{array}\right) .
$$

After solving the corresponding secular equation, the dispersion dependence in the case under consideration is found in the following way:

$$
E(\mathbf{k})=a k_{x}^{2}+b k_{y}^{2}+c k_{z}^{2} \pm \sqrt{d^{2} k_{y}^{2} k_{z}^{2}}
$$




\begin{tabular}{|l|l|l|l|}
\hline \multicolumn{2}{|c|}{ Symmetry groups } & $\begin{array}{l}\text { The extreme- } \\
\text { ness in main } \\
\text { directions } \mathrm{x}, \mathrm{y}, \\
\mathrm{z}\end{array}$ & $\begin{array}{l}\text { Strains leading } \\
\text { to the symme- } \\
\text { try lowering }\end{array}$ \\
\hline Unstrained crystal & $D_{2 h}^{16}$ & $\left.\begin{array}{l}x, y, z \\
=0\end{array}\right\}$ & $\varepsilon_{i j}=0$ \\
\hline $\begin{array}{l}\text { Strained crystal } \\
\text { simulated by } \\
\text { mathematical } \\
\text { deformation }\end{array}$ & $C_{2 h}^{2}$ & $\left\{\begin{array}{l}x, y=0 \\
z \neq 0\end{array}\right\}$ & $\varepsilon_{x y}$ \\
\cline { 2 - 4 } & $C_{2 h}^{5}$ & $\left.\begin{array}{l}x, z=0 \\
y \neq 0\end{array}\right\}$ & $\varepsilon_{x z}$ \\
\cline { 2 - 4 } & $C_{2 h}^{5}$ & $\left\{\begin{array}{l}x, y, z \\
=0\end{array}\right\}$ & $\varepsilon_{y z}$ \\
\hline
\end{tabular}

Table 1. The extremeness of $E(\mathbf{k})$ in the T point in main directions of the Brillouin zone [17].

\begin{tabular}{|l|l|l|l|}
\hline$\tau^{s}(g)$ & $A_{i s}$ & \multicolumn{2}{|c|}{$f_{i s}^{r}(k, \tilde{\varepsilon})$} \\
\cline { 3 - 4 } & & $f=1$ & $f=-1$ \\
\hline$\Gamma_{1}$ & $\left(\begin{array}{ll}1 & 0 \\
0 & 1\end{array}\right)$ & $k_{x}^{2}, k_{y}^{2}, k_{z}^{2}$ & - \\
\hline$\Gamma_{2}$ & $\left(\begin{array}{ll}1 & 0 \\
0 & -1\end{array}\right)$ & - & $\varepsilon_{x y} k_{z}\left(\varepsilon_{x z} k_{y}\right)$ \\
\hline$\Gamma_{3}$ & $\left(\begin{array}{ll}0 & 1 \\
1 & 0\end{array}\right)$ & $k_{y} k_{z}$ & - \\
\hline
\end{tabular}

Table 2. The $A_{i s}$ matrices and the $f_{i s}^{r}(\mathbf{k}, \tilde{\varepsilon})$ basis functions for the $\mathrm{T}$ point in the presence of $\varepsilon_{x y}\left(\varepsilon_{x z}\right)$ shear strains. 

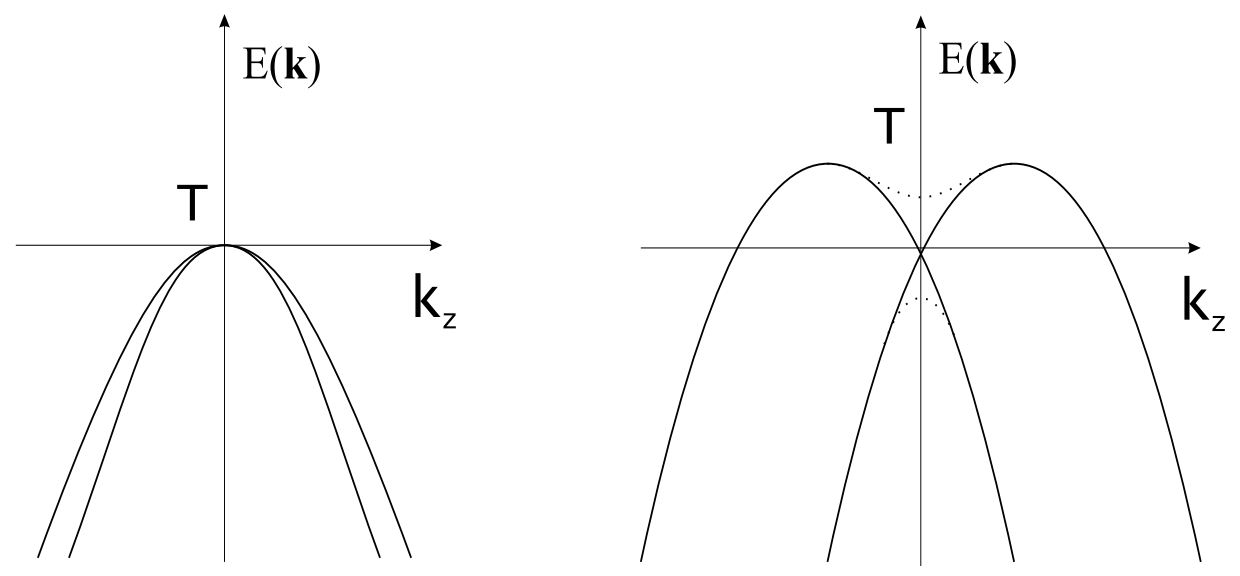

Figure 2. The band topology changes in the vicinity of the highly symmetrical $\mathrm{T}$ point caused by external shear strains [17]: left - unstrained crystal; right strained crystal.

When the external shear strain $\varepsilon_{x y}$ is present, the $D(\mathbf{k})$ matrix takes the form:

$$
D(\mathbf{k})=\left(\begin{array}{ll}
a k_{x}^{2}+b k_{y}^{2}+c k_{z}^{2}+f \varepsilon_{x y} k_{z} & d k_{y} k_{z} \\
d k_{y} k_{z} & a k_{x}^{2}+b k_{y}^{2}+c k_{z}^{2}-f \varepsilon_{x y} k_{z}
\end{array}\right),
$$

and a k-linear term appears in the corresponding dispersion law:

$$
E(\mathbf{k})=a k_{x}^{2}+b k_{y}^{2}+c k_{z}^{2} \pm \sqrt{f^{2} \varepsilon_{x y}^{2} k_{z}^{2}+d^{2} k_{y}^{2} k_{z}^{2}} .
$$

The dispersion law containing a $\mathbf{k}$-linear term in the vicinity of the $\mathrm{T}$ point in the presence of the shear strain $\varepsilon_{x z}$ is obtained in the same way.

An analysis of the dispersion law (4) shows that within the framework of the chosen approximation with the accuracy up to $k^{3}\left(\varepsilon_{x y}\left(\varepsilon_{x z}\right)\right.$ is of $k^{2}$ order $)$ there is no splitting of the twofold degenerate energy state and two extrema of $E(\mathbf{k})$ symmetrically shifted in the $k_{z}\left(k_{y}\right)$ direction appear in the vicinity of the $\mathrm{T}$ point (figure 2).

So, we can make an important conclusion that the appearance of the $\mathbf{k}$-linear terms in the energy spectrum of the SbSI crystal caused by the shear strains $\varepsilon_{x y}$ $\left(\varepsilon_{x z}\right)$ results in the shifts of the extrema from the $\mathrm{T}$ point in $k_{z}\left(k_{y}\right)$ direction. It should be noted that such shifts caused by a spin-orbit interaction are observable in the crystals with a lack of inversion symmetry. The existence of the $\mathbf{k}$-linear terms in the dispersion law of the SbSI crystal under the shear strain effect can be manifested in strong electric fields when the charge carriers heating takes place (such effects are observable in Te with the energy spectrum containing a k-linear term [18]), as well as in optical absorption spectra and in other physical effects when the peculiarities of the energy band structure are very important. Moreover, a possibility of managing such k-linearities is predictable. It is worth noting that in the SbSI crystal, as it is indicated in [19], both in the paraelectric and in the ferroelectric phase, an elastic modulus $\mathrm{C}_{66}$ is the smallest one and it is four times less in magnitude than the $\mathrm{C}_{44}$ one. That is why $\varepsilon_{x y}$ will be the largest component of the tensor of deformation under 
the same strains. This leads to the possibility of considering $\varepsilon_{x y} k_{z}$ to be the value proportional to $\mathbf{k}^{2}$ in contrast to weakly anisotropic crystals. Adding a symmetrical term proportional to $\varepsilon_{x y} \varepsilon_{x z}$ to the off-diagonal elements in $D(\mathbf{k})$ we shall obtain the splitting within the same approximation. This causes a warped dependence of the upper valence band (dotted line in figure 2, right) with the negative effective mass. The same splitting can be obtained when the inhomogeneous bonding between structural units is taken into account and is accompanied by symmetry lowering after the mathematical deformation. But it can be observed when the components of higher order of magnitude are taken into account.

K.Z.R. is very grateful to Professor M.Scheffler for his permission to utilize the fhi98md and fhi98PP computer program.

\section{References}

1. Nakao K., Balkanski M. Electronic band structures of SbSI in the para- and ferroelectric phases. // Phys. Rev. B, 1973, vol. 8, No. 12, p. 5759-5780.

2. Fong C.Y., Petroff Y., Kohn S., Shen Y.R. Wavelength modulation spectra of SbSI, its electronic band structure. // Solid State Commun, 1974, vol. 14, No. 8, p. 681-685.

3. Bercha D.M., Mitin O.B. A complexation in energy spectrum of rhombic semiconductors as a consequence of symmetry and strong anisotropy. // Fiz. Tekh. Polup., 1987, vol. 21, No. 8, p. 1508-1512.

4. Fridkin V.M. Ferroelectrics-Semiconductors. Moscow, Nauka, 1976 (in Russian).

5. Dönges E. Über Thiohalogenide des dreiwertigen Antimons und Wismuts. // Z. anorg. und allg. Chem. B, 1950, vol. 263, p. 112-132.

6. Ihm J., Zunger A., Cohen M.L. Momentum-space formalism for the total energy of solids. // J. Phys. C, 1979, vol. 12, No. 21, p. 4409-4422.

7. Hamann D.R. Generalized norm-conserving pseudopotentials. // Phys. Rev. B, 1989, vol. 40, No. 20, p. 2980-2987.

8. Fuchs M., Scheffler M. Ab initio pseudopotentials for electronic structure calculations of poly-atomic systems using density-functional theory. // Comp. Phys. Commun., 1998, vol. 119, p. 67.

9. Ceperley M., Alder B.J. Ground state of the electron gas by a stochastic method. // Phys. Rev. Lett., 1980, vol. 45, No. 7, p. 566-569.

10. Perdew J.P., Zunger A. Self-interaction correction to density-functional approximations for many-electron systems. // Phys. Rev. B, 1981, vol. 23, No. 10, p. 5048-5079.

11. Gonze X., Stumpf R., Scheffler M. Analysis of separable potentials. // Phys. Rev. B, 1991, vol. 44, No. 16, p. 8503-8513.

12. Bockstedte M., Kley A., Neugebauer J., Scheffler M. Density-functional theory calculations for poly-atomic systems: electronic structure, static and elastic properties and ab initio molecular dynamics. // Comp. Phys. Commun., 1997, vol. 107, p. 187.

13. Monkhorst H.J., Pack J.D. Special points for Brillouin-zone integrations. // Phys. Rev. B, 1976, vol. 13, No. 12, p. 5188-5192.

14. Alward J.W., Fong C.Y., El-Batomouny M., Wollen F. Electronic and optical properties of SbSBr, SbSI and SbSeI. // Solid State Commun., 1978, vol. 25, p. 307-310.

15. Bercha D.M., Nebola I.I., Bercha I.V. A correlation between chains and energy spectrum of the chained crystals. // Fiz. Tverd. Tela, 1978, vol. 20, No. 5, p. 1320-1325 
(in Russian).

16. Pikus G.E. A new method of calculations of the charge carriers energy spectra. // Zh. Theoret. Eksp. Fiz., 1961, vol. 41, No. 4, p. 1258-1273 (in Russian).

17. Bercha I.V. Deformational effects in crystals with distinguished structural units. - In: Complicated semiconductors (obtaining, properties and applying). Uzhgorod, 1981, p. 143-150 (in Russian).

18. Gorley P.N., Shenderowskii V.A. The Variational Method in the Kinetic Theory. Kyiv, Naukowa dumka, 1992.

19. Sandercock S.B. Brillouin scattering study of SbSI using a double-passed, stabilized scanning interferometer. // Opt. Commun., 1970, vol. 2, No. 2, p. 73-76.

\title{
Відображення впливу деформацій в зонних спектрах в кристалах з неоднорідним зв'язком
}

\author{
Д.М.Берча ${ }^{1,2}$, К.З.Рущанський ${ }^{1}$, І.В.Сліпухіна ${ }^{1}$, \\ I.В.Берча ${ }^{1}$ \\ 1 Ужгородський національний університет, \\ 88000 Ужгород, вул. Волошина, 54 \\ 2 Інститут фізики, Педагогічний університет, \\ Польща, 35-310 Жешув, вул. Рейтана, 16а
}

Отримано 25 жовтня 2002 р., в остаточному вигляді - 3 квітня 2003 p.

Теоретико-груповим методом в поєднанні з методом інваріантів Пікуса досліджено вплив зсувових деформацій на енергетичний спектр сильно анізотропного кристала SbSI. Для визначення зонної структури кристала було застосовано розрахунки з перших принципів з використанням локального наближення. Дослідження зонної структури вказали на точну локалізацію двократно виродженого максимума валентної зони в точці Т. Виявилося, що зсувові деформації приводять до зміни топології зон в околі точки Т, а у відповідному законі дисперсії з'являється k -лінійний член.

Ключові слова: ab initio, деформації, SbSI

PACS: 71.15.Ap, 71.15. $\mathrm{Hx}, 71.15 . \mathrm{Mb}, 61.50 . \mathrm{Ah}$ 